\title{
Associate, Hyperdomainlike, and Presimplifiable Hyperrings
}

\author{
Agboola Adesina Abdul Akeem ${ }^{1}$ and Davvaz Bijan ${ }^{2}$ \\ ${ }^{1}$ Department of Mathematics, Federal University of Agriculture, PMB 2240, Abeokuta, Ogun State, Nigeria \\ ${ }^{2}$ Department of Mathematics, Yazd University, P.O. Box 89195-741, Yazd, Iran
}

Correspondence should be addressed to Agboola Adesina Abdul Akeem; aaaola2003@yahoo.com

Received 12 February 2014; Accepted 17 June 2014; Published 14 August 2014

Academic Editor: Feng Feng

Copyright (C) 2014 A. Adesina Abdul Akeem and D. Bijan. This is an open access article distributed under the Creative Commons Attribution License, which permits unrestricted use, distribution, and reproduction in any medium, provided the original work is properly cited.

Based on the works of Axtell et al., Anderson et al., and Ghanem on associate, domainlike, and presimplifiable rings, we introduce new hyperrings called associate, hyperdomainlike, and presimplifiable hyperrings. Some elementary properties of these new hyperrings and their relationships are presented.

\section{Introduction}

The study of strongly associate rings began with Kaplansky in [1] and was further studied in [2-5]. Domainlike rings and their properties were presented by Axtell et al. in [6]. Presimplifiable rings were introduced by Bouvier in the series of papers [7-11] and were later studied in [2-4]. Further properties of associate and presimplifiable rings were recently presented by Ghanem in [12].

The theory of hyperstructures was introduced in 1934 by Marty [13] at the 8th Congress of Scandinavian Mathematicians. Introduction of the theory has caught the attention and interest of many mathematicians and the theory is now spreading like wild fire. The notion of canonical hypergroups was introduced by Mittas [14]. Some further contributions to the theory can be found in [15-19].

Hyperrings are essentially rings with approximately modified axioms. Hyperrings $(R,+, \cdot)$ are of different types introduced by different researchers. Krasner [20] introduced a type of hyperring $(R,+, \cdot)$ where + is a hyperoperation and $\cdot$ is an ordinary binary operation. Such a hyperring is called a Krasner hyperring. Rota in [21] introduced a type of hyperring $(R,+, \cdot)$ where + is an ordinary binary operation and $\cdot$ is a hyperoperation. Such a hyperring is called a multiplicative hyperring. de Salvo [22] introduced and studied a type of hyperring $(R,+, \cdot)$ where + and $\cdot$ are hyperoperations. The most comprehensive reference for hyperrings is Davvaz and
Leoreanu-Fotea's book [18]. Some other references are [2331].

In this paper, we present and study associate, hyperdomainlike, and presimplifiable hyperrings. The relationships between these new hyperrings are presented.

\section{Preliminaries}

In this section, we will provide some definitions that will be used in the sequel. For full details about associate, domainlike, and presimplifiable rings, the reader should see $[1,4-$ $6,12]$. Also, for details about hyperstructures and hyperrings, the reader should see [12].

Definition 1. Let $R$ be a commutative ring with unity.

(1) $R$ is called an associate ring if whenever any two elements $x, y \in R$ generate the same principal ideal of $R$, there is a unit $u \in U(R)$ such that $x=u y$.

(2) $R$ is called a domainlike ring if all zero divisors of $R$ are nilpotent.

(3) $R$ is called a presimplifiable ring if, for any two elements $x, y \in R$ with $x=x y$, we have $x=0$ or $y \in U(R)$.

(4) $R$ is called a superassociate ring if every subring of $R$ is associate. 
(5) $R$ is called a superpresimplifiable ring if every subring of $R$ is presimplifiable.

Example 2. Integral domains, domainlike, and local rings are presimplifiable rings and hence are associate rings.

Example 3 (see [6]). It is easy to check that $\mathbb{Z}_{n}$ is presimplifiable if and only if $n=p^{m}$, where $p$ is some prime. Hence, $\mathbb{Z}_{n}$ is presimplifiable if and only if $\mathbb{Z}_{n}$ is local. Also, if a ring $R$ is quasilocal, then $R$ is presimplifiable, since $J(R)=M$, the unique maximal ideal of $R$, and thus $Z(R) \subseteq M=J(R)$.

Definition 4. Let $H$ be a nonempty set and let $\circ$ : $H \times$ $H \rightarrow \mathscr{P}^{*}(H)$ be a hyperoperation, where $\mathscr{P}^{*}(H)$ is the family of all nonempty subsets of $H$. The couple $(H, \circ)$ is called a hypergroupoid. For any two nonempty subsets $A$ and $B$ of $H$ and $x \in H$, one defines

$$
\begin{gathered}
A \circ B=\bigcup_{a \in A, b \in B} a \circ b, \\
A \circ x=A \circ\{x\}, \\
x \circ B=\{x\} \circ B .
\end{gathered}
$$

Definition 5. A hypergroupoid $(H, \circ)$ is called a semihypergroup if for all $a, b, c$ of $H$ one has $(a \circ b) \circ c=a \circ(b \circ c)$, which means that

$$
\bigcup_{u \in a \circ b} u \circ c=\bigcup_{v \in b \circ c} a \circ v .
$$

A hypergroupoid $(H, \circ)$ is called a quasihypergroup if for all $a$ of $H$ one has $a \circ H=H \circ a=H$. This condition is also called the reproduction axiom.

A hypergroupoid $(H, \circ)$ which is both a semihypergroup and a quasihypergroup is called a hypergroup.

Definition 6. Let $H$ be a nonempty set and let + be a hyperoperation on $H$. The couple $(H,+)$ is called a canonical hypergroup if the following conditions hold:

(1) $x+y=y+x$, for all $x, y \in H$;

(2) $x+(y+z)=(x+y)+z$, for all $x, y, z \in H$;

(3) there exists a neutral element $0 \in H$ such that $x+0=$ $\{x\}=0+x$, for all $x \in H$

(4) for every $x \in H$, there exists a unique element $-x \in H$ such that $0 \in x+(-x) \cap(-x)+x$;

(5) $z \in x+y$ implies $y \in-x+z$ and $x \in z-y$, for all $x, y, z \in H$.

Definition 7. Let $R$ be a nonempty set and let + and $\cdot$ be hyperoperation and usual operation on $R$, respectively. The triple $(R,+, \cdot)$ is called a Krasner hyperring if the following axioms hold:

(1) $(R,+)$ is a canonical hypergroup;

(2) $(R, \cdot)$ is a semigroup having zero as a bilaterally absorbing element; that is, $x \cdot 0=0 \cdot x=0$ for all $x \in R$;
(3) multiplication - is distributive over the hyperoperation + ; that is, $x \cdot(y+z)=x \cdot y+x \cdot z$ and $(y+z) \cdot x=$ $y \cdot x+z \cdot x$ for all $x, y, z \in R$.

If $(R,+, \cdot)$ is a hyperring, $A$ and $B$ are nonempty subsets of $R$ and $x \in R$; then we define

$$
\begin{gathered}
A+B=\{y: y \in a+b \text { for some } a \in A, b \in B\}, \\
A \cdot B=\left\{y: y \in \sum_{i=1}^{n} a_{i} b_{i}, a_{i} \in A, b_{i} \in B, n \in \mathbb{Z}^{+}\right\}, \\
A+x=A+\{x\} \\
x+B=\{x\}+B, \\
-A=\{-a: a \in A\} .
\end{gathered}
$$

A hyperring $(R,+, \cdot)$ is called a commutative hyperring with unity if $(R, \cdot)$ is a commutative semigroup with unity.

For any $x, y \in R$, we use $x y$ instead of $x \cdot y$.

\section{Associate, Domainlike, and Presimplifiable Hyperrings}

Throughout this section, all hyperrings will be assumed to be commutative Krasner hyperrings with unity.

$J(R), N(R), U(R)$, and $Z(R)$ will denote Jacobson radical, nilradical, the set of units, and the set of zero divisors of $R$, respectively. $\operatorname{Ann}(x)$ will denote the annihilator of $0 \neq x \in R$.

Let $X$ be a subset of a hyperring $R$. Let $\left\{A_{i} \mid i \in J\right\}$ be the family of all hyperideals in $R$ which contain $X$. Then, $\bigcap_{i \in J} A_{i}$ is called the hyperideal generated by $X$. This hyperideal is denoted by $\langle X\rangle$. If $X=\left\{x_{1}, \ldots, x_{n}\right\}$, then the hyperideal $\langle X\rangle$ is denoted by $\left\langle x_{1}, \ldots, x_{n}\right\rangle$.

Lemma 8 (see [18]). Let $R$ be a hyperring and $a \in R$. Then,

(1) $R a=a R$ is a hyperideal of $R$;

(2) if $R$ has a unit element, then $R a=\langle a\rangle=a R$.

Definition 9. Let $R$ be a hyperring and let $\rho_{\sim}, \rho_{\approx}$, and $\rho_{\cong}$ be relations on $R$ defined as follows.

(1) $x \rho_{\sim} y$ if and only if $\langle x\rangle=\langle y\rangle$, for all $x, y \in R$.

(2) $x \rho_{\approx} y$ if and only if $x=u y$, for all $x, y \in R$ and some $u \in U(R)$.

(3) $x \rho_{\cong} y$ if and only if $x \rho_{\sim} y$ and when $x \neq 0$ and $x=r y$, then $r \in U(R)$.

Theorem 10. Let $R$ be a hyperring. Then,

(1) $\rho_{\sim}$ is an equivalence relation on $R$,

(2) $\rho_{\approx}$ is an equivalence relation on $R$,

(3) $\rho_{\cong}$ is an equivalence relation on $R$ if and only if, for all $x, y \in R, x=x y$ implies $x=0$ or $y \in U(R)$. 
Proof. (1) and (2) are clear. For (3), suppose that $\rho_{\cong}$ is an equivalence on $R$ and suppose that $x=x y$ with $x \neq 0$. Then, $x a=0$ for some $a \in 1-y$ which implies $y \in 1-a$. But then, $a=0$ so that $y \in U(R)$. The converse is straightforward.

Definition 11. If $R$ is a hyperring, then $a \neq 0$ in $R$ is said to be a zero divisor if there exists $b \neq 0$ in $R$ such that $a b=0$.

Definition 12. A hyperring is a hyperdomain if it has no zero devisor.

The following example is presented in $[18,29,30]$.

Example 13. Let $(G, \cdot)$ be a finite group with $m$ elements, $m>$ 3 , and define a hyperaddition and a multiplication on $H=$ $G \cup\{0\}$, by

$$
\begin{gathered}
a+0=0+a=\{a\}, \quad \forall a \in H, \\
a+a=\{a, 0\}, \quad \forall a \in G, \\
a+b=b+a=H \backslash\{a, b\}, \quad \forall a, b \in G, a \neq b, \\
a \odot 0=0, \quad \forall a \in H, \\
a \odot b=a \cdot b, \quad \forall a, b \in G .
\end{gathered}
$$

Then, $(H,+, \odot)$ is a hyperring.

Example 14. It is easy to see that Example 13 is a hyperdomain.

Theorem 15. Let $R$ be a hyperring.

(1) $x \rho_{\cong} y$ implies $x \rho_{\sim} y$, for all $x, y \in R$.

(2) $x \rho_{\approx} y$ implies $x \rho_{\sim} y$, for all $x, y \in R$.

(3) If $R$ is hyperdomain, then $x \rho_{\sim} y$ implies $x \rho_{\approx} y$, for all $x, y \in R$.

Proof. (1) and (2) are obvious. For (3), suppose that $R$ is hyperdomain and suppose that $x \rho_{\sim} y$. Then, $\langle x\rangle=\langle y\rangle$ which implies that $x=k_{1} y$ and $y=k_{2} x$ for some $k_{1}, k_{2} \in R$. Thus, $x=k_{1} k_{2} x$ which implies $x a=0$ for some $a \in 1-k_{1} k_{2} \subseteq R$ so that $k_{1} k_{2} \in 1-a$. Since $R$ is a hyperdomain, if $x \neq 0$, then $a=0$, and, therefore, $k_{1} k_{2} \in U(R)$ which implies $k_{1}, k_{2} \in U(R)$. Hence, $x \rho_{\approx} y$.

Definition 16. Let $R$ be a hyperring.

(1) $R$ is said to be an associate hyperring if $x \rho_{\sim} y$ implies $x \rho_{\approx} y$ for all $x, y \in R$.

(2) $R$ is said to be a superassociate hyperring if every subhyperring of $R$ is associate.
Example 17. The hyperring in Example 13 is associate hyperring and superassociate hyperring too.

Definition 18. Let $R$ be a hyperring.

(1) $R$ is said to be a presimplifiable hyperring if, for all $x, y \in R, x=x y$ implies $x=0$ or $y \in U(R)$.

(2) $R$ is said to be a superpresimplifiable hyperring if every subhyperring of $R$ is presimplifiable.

(3) $R$ is said to be a hyperdomainlike hyperring if all zero divisors of $R$ are nilpotent.

Example 19. Let $R_{1}=\mathbb{Z}_{p^{m}}, R_{2}=\mathbb{Z}_{p^{n}}$, and $R_{3}=\mathbb{Z}_{p^{k}}$ be three rings, where $m, n>k$ and $p$ is a prime number. Suppose that $M_{i}, i=1,2,3$, are maximal ideals of $R_{i}, i=1,2,3$. Let $H_{i}$, $i=1,2,3$, be subsets of $R_{i}$ and define a hyperaddition and multiplication on $H_{i}$ by

$$
\begin{gathered}
x \underset{i}{\oplus} y=x+y+M_{i}, \quad i=1,2,3, \forall x, y \in H_{i}, \\
x \odot y=x y, \quad \forall x, y \in H_{i} .
\end{gathered}
$$

Then, $\left(H_{i}, \oplus_{i}, \odot\right)$ are hyperrings. Since $R_{i}, i=1,2,3$, are domainlike (see Example 3), consequently each $H_{i}$ is hyperdomainlike.

Theorem 20. Let $R$ be a hyperring. The following conditions are equivalent:

(1) $x \rho_{\sim} y$ implies $x \rho_{\cong} y$, for all $x, y \in R$;

(2) $x \rho_{\approx} y$ implies $x \rho_{\cong} y$, for all $x, y \in R$;

(3) $x \rho_{\cong} x$ for all $x \in R$;

(4) $R$ is presimplifiable;

(5) $Z(R) \subseteq 1-U(R)$;

(6) $Z(R) \subseteq J(R)$;

(7) for $0 \neq x \in R, y\langle x\rangle=\langle x\rangle$ implies $y \in U(R)$.

Proof. $(1 \Rightarrow 2)$ : it follows from the definitions of $\rho_{\sim}, \rho_{\approx}$, and $\rho_{\cong}$.

$(2 \Rightarrow 3)$ : suppose that $x \rho_{\approx} y$ which implies that $x \rho_{\cong} y$ for all $x, y \in R$. Then, $x \rho_{\approx} x$ implies $x=1 \cdot x$ and, therefore, $x \rho_{\cong} x$ for all $x \in R$.

(3 $\Rightarrow 4$ ): suppose that $x \rho_{\cong} x$ for all $x \in R$ and suppose that $x=x y$. Then, $x a=0$ for some $a \in 1-y$ which implies $y \in 1-a \subseteq R$. If $x \neq 0$, we must have $y \in U(R)$. Hence, $R$ is presimplifiable.

( $4 \Rightarrow 5$ ): suppose that $R$ is presimplifiable and suppose that $x \in Z(R)$. Then, there exists $0 \neq y \in R$ such that $x y=0$. But then, $y \in y-x y$ which implies that $y=y a$ for some $a \in 1-x \subseteq U(R)$ so that $x \in 1-U(R)$.

$(5 \Rightarrow 6)$ : suppose that $x \in Z(R)$. Then, $y=x r \in Z(R)$ for all $0 \neq r \in R$. Thus, $y \in 1-u$ for some $u \in U(R)$. Now, $u \in 1-y$ implies $1-y \subseteq U(R)$ and, therefore, $x \in J(R)$.

$(6 \Rightarrow 7)$ : suppose that $0 \neq x \in R$ and $y\langle x\rangle=\langle x\rangle$. Then, $x=y x r$ for some $r \in R$ so that $x a=0$ for some $a \in 1-y r$. 
Since $x \neq 0$, we must have $a \in Z(R) \subseteq J(R)$ so that $y r \in$ $1-a \subseteq U(R)$ and, therefore, $y \in U(R)$.

$(7 \Rightarrow 1)$ : suppose that $x \rho_{\sim} y$ and $x \neq 0$. Then, $\langle x\rangle=\langle y\rangle$ and $x=r y$ for some $r \in R$. Thus, $x\langle x\rangle=r y\langle y\rangle=r\langle y\rangle$ and we have $\langle x\rangle=r\langle x\rangle$ which implies $r \in U(R)$. Hence, $x \rho_{\cong} y$.

Theorem 21. Let $R$ be a hyperring. If $R$ is quasilocal, then it is presimplifiable.

Proof. Suppose that $R$ is quasilocal. Then, $R$ has a unique maximal hyperideal $M$ and $J(R)=M$. Hence, $Z(R) \subseteq M=$ $J(R)$ and therefore $R$ is presimplifiable.

Theorem 22. Let $R$ be a hyperring. If $R$ is presimplifiable, then $R$ is associate.

Proof. It follows from Theorem 20.

Corollary 23. Any hyperdomain or any quasilocal hyperring is an associate hyperring.

Proof. This is immediate from Theorems 20, 21, and 22.

Theorem 24. Let $R$ be a hyperring. Then, the following statements are equivalent:

(1) $R$ is hyperdomainlike;

(2) $Z(R) \subseteq N(R)$;

(3) $\langle 0\rangle$ is a primary hyperideal of $R$.

Proof. $(1 \Rightarrow 2)$ : it is obvious.

$(2 \Rightarrow 3)$ : let $x y \in\langle 0\rangle$. Then, $x y=0$. If $y \neq 0$, then $x \in Z(R) \subseteq N(R)$ so that $x^{n}=0$ for some positive integer $n$ and, therefore, $x^{n} \in\langle 0\rangle$. Hence, $\langle 0\rangle$ is primary.

(3) implies (1): suppose that $\langle 0\rangle$ is a primary hyperideal of $R$. Let $x \in Z(R)$ be arbitrary. Then, there exists $0 \neq y \in$ $R$ such that $x y=0$ which implies that $x y \in\langle 0\rangle$. Since $y \notin\langle 0\rangle$, we must have $x^{n} \in\langle 0\rangle$ and therefore $x^{n}=0$. Hence, $x$ is nilpotent. Since $x$ is arbitrary, it follows that $R$ is hyperdomainlike.

Let $R_{1}$ and $R_{2}$ be any two hyperrings and let $R=R_{1} \times R_{2}$. For any $(a, b),(c, d) \in R$, define

$$
\begin{gathered}
(a, b)+(c, d)=\{(x, y): x \in a+c, y \in b+d\}, \\
(a, b) \cdot(c, d)=(a \cdot c, b \cdot d) .
\end{gathered}
$$

Then, $(R,+, \cdot)$ is a hyperring called the direct product of $R_{1}$ and $R_{2}$.

Theorem 25. Let $R$ be a hyperring.

(1) If $R$ is hyperdomainlike, then it is superpresimplifiable.

(2) If $R$ is presimplifiable (associate), then a subhyperring of $R$ need not be presimplifiable (associate).
(3) If $R$ is superassociate, then it need not be presimplifiable.

(4) A direct product of superassociate hyperrings need not be superassociate.

Proof. See Anderson et al. [4].

Theorem 26. Let $R_{1}$ and $R_{2}$ be any two presimplifiable hyperrings with the set of units $\{ \pm 1\}$. Then, $R=R_{1} \times R_{2}$ is a superassociate hyperring.

Proof. The proof is similar to the proof of Theorem 2.1 in [12].

Theorem 27. Let $A$ be a hyperideal of a hyperring R. $A$ is primary if and only if $R / A$ is hyperdomainlike.

Proof. Suppose that $A$ is primary. Let $\bar{x} \in Z(R / A)$. Then, there exists $\overline{0} \neq \bar{y} \in R / A$ such that $\bar{x} \bar{y}=\overline{0}$ which implies that $x y+A \subseteq A$ which implies $x y \in A$. Since $A$ is primary and $y \notin A$, it follows that $x^{n} \in A$ from which we have $\bar{x}^{n}=\overline{0}$ and therefore $\bar{x} \in N(R / A)$. Hence, $Z(R / A) \subseteq N(R / A)$ and, thus, $R / A$ is hyperdomainlike.

Conversely, suppose that $R / A$ is hyperdomainlike. Since $A$ is a zero of $R / A$, it follows that $A$ is primary.

Theorem 28. Let $R$ be a hyperring. If $R$ is hyperdomainlike, then $R$ is presimplifiable.

Proof. The proof follows from Theorem 21, since $Z(R) \subseteq$ $N(R) \subseteq J(R)$.

Theorem 29. Let $R$ be a hyperring. If $R$ is hyperdomainlike, then $R / N(R)$ is a hyperdomain.

Proof. Suppose that $R$ is hyperdomainlike. Let $\bar{x}, \bar{y} \in R / N(R)$ such that $\bar{x} \bar{y}=\overline{0}$ with $\overline{0} \neq \bar{x}$. Then, $x y+N(R) \subseteq N(R)$ implies $x y \in N(R)$ but $x \notin N(R)$. For some positive integer $n$, we have $(x y)^{n}=0$ which implies $x^{n} y^{n} \in\langle 0\rangle$. Since $x^{n} \notin\langle 0\rangle$, we must have $\left(y^{n}\right)^{k} \in\langle 0\rangle$ for some positive integer $k$. Hence, $y \in N(R)$ and, therefore, $\bar{y}=\overline{0}$. Hence, $R / N(R)$ is a hyperdomain.

Theorem 30. Let $R$ be a hyperring. If $R$ is hyperdomainlike, then $Z(R)$ is the unique minimal prime hyperideal of $R$.

Proof. Suppose that $R$ is hyperdomainlike. Then, $Z(R) \subseteq$ $N(R)$. Since $N(R)$ is the intersection of all prime hyperideals of $R$, the required result follows.

Theorem 31. Let $R$ be a hyperring and let $A$ be a hyperideal of $R$. Then, $R / r(A)$ is hyperdomainlike if and only if $R / r(A)$ is a hyperdomain.

Proof. Suppose that $R / r(A)$ is a hyperdomain. Then, $r(A)$ is a prime hyperideal of $R$ which is clearly a primary hyperideal of $R / r(A)$. Hence, $R / r(A)$ is hyperdomainlike.

Conversely, suppose that $R / r(A)$ is hyperdomainlike. Let $\bar{x}, \bar{y} \in R / r(A)$ such that $\bar{x} \bar{y}=\overline{0}$ with $\overline{0} \neq \bar{x}$. Then, $x y+r(A) \subseteq$ $r(A)$ implies $x y \in r(A)$ but $x \notin r(a)$. Since $r(A)$ is primary in 
$R / r(A)$, we must have $y^{n} \in r(A)$ for some positive integer $n$. Thus, $\left(y^{n}\right)^{k} \in A$ for some positive integer $k$. Thus, $y \in r(A)$ and, therefore, $\bar{y}=\overline{0}$. Hence, $R / r(A)$ is a hyperdomain.

Theorem 32. Let $R$ be a hyperring. $R / N(R)$ is a hyperdomainlike ring if and only $R / N(R)$ is a hyperdomain.

Proof. The proof is similar to the proof of Theorem 29.

Theorem 33. Let $R$ be a hyperring and let $S^{-1} R$ be the total quotient hyperring of $R$. If $R$ is hyperdomainlike so also is $S^{-1} R$.

Proof. The proof is similar to the classical ring and therefore omitted.

Theorem 34. Let $R$ be a hyperring. $R / N(R)$ is presimplifiable if and only if whenever $x=x y$ and $x \notin N(R)$, then $y \in U(R)$.

Proof. Suppose that $R / N(R)$ is presimplifiable and suppose that $x=x y$ with $x \notin N(R)$. Then, $\bar{x}=\bar{x} \bar{y}$ and $\bar{x} \neq \overline{0}$ so that $\bar{y} \in U(R / N(R))$. Now, $x-x y \subseteq N(R)$ which implies that $x a \in N(R)$ for some $a \in 1-y$ and therefore $x^{n} a^{n}=0$ and $a^{n} \in 1-y b$, where $b \in \sum_{r=1}^{n}(-1)^{r-1}\left(\begin{array}{c}n \\ r\end{array}\right) y^{r-1}$ and $y b \in 1-a^{n}$ so that $x^{n} y b \in x^{n}-x^{n} a^{n}$. Since $x^{n} \neq 0$, we must have $y b \in U(R)$ and hence $y \in U(R)$.

Conversely, suppose that $x=x y$ with $x \notin N(R)$ implies that $y \in U(R)$. Then, $\bar{x}=\bar{x} \bar{y}$ and $\bar{x} \neq \overline{0}$. It can be shown that $\bar{y} \in U(R / N(R))$. Hence, $R / N(R)$ is presimplifiable.

Corollary 35. Let $R$ be a hyperring. If $R$ is presimplifiable, then $R / N(R)$ is presimplifiable and hence strongly associate.

Definition 36. Let $A$ be a hyperideal of a hyperring $R$ and let $A^{*}=A-\{0\} . A$ is called a presimplifiable hyperideal if, for every $x \in A^{*}$ and $y \in R, x=x y$ implies that $y \in U(R)$.

Lemma 37. Every hyperideal of a presimplifiable hyperring $R$ is presimplifiable.

Proof. It is obvious.

Theorem 38. Let $A$ be a hyperideal of a hyperring $R$. Then, $A$ is presimplifiable if and only if $A n n(x) \subseteq J(R)$ for all $x \in A^{*}$.

Proof. Suppose that $A$ is presimplifiable. Let $y \in \operatorname{Ann}(x)$, where $x \in A^{*}$. Then, $x y=0$ and we can write $x=x a$ for some $a \in 1-r y$ and $r \in R$. Since $R$ is presimplifiable, we have $a \in U(R)$ so that $1-r y \subseteq U(R)$. Hence, $y \in J(R)$.

Conversely, suppose that $\operatorname{Ann}(x) \subseteq J(R)$ for all $x \in A^{*}$. Let $x=x y$. Then, $x a=0$ for some $a \in 1-y$. Thus, $a \in$ $\operatorname{Ann}(x) \subseteq J(R)$ and, therefore, $1-y \in J(R)$ so that $y \in U(R)$. Hence, $A$ is presimplifiable.

Theorem 39. Let $R$ be a hyperring. Then, $R$ is presimplifiable if and only if $R / N(R)$ and $N(R)$ are presimplifiable.

Proof. Suppose that $R$ is presimplifiable. Let $\bar{x} \in Z(R / N(R))-$ $\overline{0}$. Then, there exits $\overline{0} \neq y \in Z(R / N(R))-\{\overline{0}\}$ such that $\bar{x} \bar{y}=\overline{0}$ so that $x y+N(R) \subseteq N(R)$ which implies that $x y \in N(R)$ and, for a positive integer $n$, we have $x^{n} y^{n}=0$. Since $y^{n} \neq 0$, we must have $x^{n} \in Z(R)$ and so $x \in Z(R) \subseteq J(R)$. Hence, $\bar{x} \in J(R / N(R))$ and, therefore, $R / N(R)$ is presimplifiable.

Conversely, suppose that $R / N(R)$ and $N(R)$ are presimplifiable. Let $0 \neq x, y \in R$ such that $x=x y$. Then, $\bar{x}=\bar{x} \bar{y}$. Obviously, $x \in N(R)$ and $\bar{y} \in U(R / N(R))$. Consequently, $y \in U(R)$ and, therefore, $R$ is presimplifiable.

Definition 40. Let $R_{1}$ and $R_{2}$ be any two hyperrings and let $\phi: R_{1} \rightarrow R_{2}$ be a mapping from $R_{1}$ into $R_{2}$.

(1) $\phi$ is called a homomorphism if

(i) $\phi(a+b) \subseteq \phi(a)+\phi(b)$, for all $a, b \in R_{1}$,

(ii) $\phi(a b)=\phi(a) \phi(b)$, for all $a, b \in R_{1}$,

(iii) $\phi(0)=0$.

(2) $\phi$ is called a good or strong homomorphism if

(i) $\phi(a+b)=\phi(a)+\phi(b)$, for all $a, b \in R_{1}$,

(ii) $\phi(a b)=\phi(a) \phi(b)$, for all $a, b \in R_{1}$,

(iii) $\phi(0)=0$.

(3) A strong homomorphism $\phi$ from a hyperring $R_{1}$ into a hyperring $R_{2}$ is called an isomorphism if $\phi$ is bijective and we write $R_{1} \cong R_{2}$.

(4) If $\phi$ is a homomorphism from a hyperring $R_{1}$ into a hyperring $R_{2}$, then the kernel of $\phi$ denoted by $\operatorname{Ker} \phi$ is the set $\left\{x \in R_{1}: \phi(x)=0\right\}$ and the image of $\phi$ denoted by $\operatorname{Im} \phi$ is the set $\left\{\phi(x): x \in R_{1}\right\}$. It is known that $\operatorname{Ker} \phi$ is a hyperideal of $R_{1}$ and $\operatorname{Im} \phi$ is a hyperideal of $R_{2}$.

Definition 41. Let $R_{1}, R_{2}$, and $R_{3}$ be any three hyperrings with strong homomorphisms $\phi_{i}: R_{i} \rightarrow R_{3}, i=1$, 2, which preserve the unity. The set $P=\left\{\left(r_{1}, r_{2}\right) \in R_{1} \times R_{2}: \phi_{1}\left(r_{1}\right)=\right.$ $\left.\phi_{2}\left(r_{2}\right)\right\}$ is called the pullback of $R_{1} \rightarrow R_{3} \leftarrow R_{2}$.

Lemma 42. Let $R_{1}, R_{2}$, and $R_{3}$ be any three hyperrings and let $P$ be the pullback of $R_{1} \rightarrow R_{3} \leftarrow R_{2}$. Then, $P$ is a subhyperring of $R_{1} \times R_{2}$ and $U(P)$, the set of units of $P$, is $\left\{\left(u_{1}, u_{2}\right) \in P: u_{1} \in\right.$ $\left.U\left(R_{1}\right), u_{2} \in U\left(R_{2}\right)\right\}$.

Theorem 43. Let $R_{1}, R_{2}$, and $R_{3}$ be any three hyperrings with strong homomorphisms $\phi_{i}: R_{i} \rightarrow R_{3}, i=1$, 2. If $\operatorname{Ker} \phi_{1} \nsubseteq$ $Z\left(R_{1}\right)$ and there exists $u \in U\left(R_{1}\right)$ with $\phi_{1}(u), \phi_{1}\left(u^{-1}\right) \in$ $\phi_{2}\left(R_{2}\right) \backslash \phi_{2}\left(U\left(R_{2}\right)\right)$, then the pullback $P$ of $R_{1} \rightarrow R_{3} \leftarrow R_{2}$ is not an associate hyperring.

Proof. The proof is similar to the proof of Proposition 6 in [4].

Theorem 44. Let $R_{1}, R_{2}$, and $R_{3}$ be any three hyperrings with strong epimorphisms $\phi_{i}: R_{i} \rightarrow R_{3}, i=1,2$, which are not strong isomorphisms. If $R_{1}$ and $R_{2}$ are hyperdomains, then the pullback $P$ of $R_{1} \rightarrow R_{3} \leftarrow R_{2}$ is associate (presimplifiable) if and only if $\phi_{1}\left(U\left(R_{1}\right)\right)=\phi_{2}\left(U\left(R_{2}\right)\right)$, respectively, $\phi_{i}^{-1}(1) \subseteq$ $U\left(R_{i}\right), i=1,2$, or, equivalently, $\phi_{i}^{-1}\left(U\left(R_{3}\right)=U\left(R_{i}\right), i=1,2\right.$. 
Proof. The proof is similar to the proof of Theorem 7 in [4].

Theorem 45. Let $R_{1}, R_{2}$, and $R_{3}$ be any three hyperrings with strong epimorphisms $\phi_{i}: R_{i} \rightarrow R_{3}, i=1,2$, which are not strong isomorphisms. If $R_{1}$ and $R_{2}$ are presimplifiable, then the pullback $P$ of $R_{1} \rightarrow R_{3} \leftarrow R_{2}$ is presimplifiable if and only if $\phi_{i}^{-1}(1) \subseteq U\left(R_{i}\right), i=1,2$.

Proof. The proof is similar to the proof of Theorem 2.3 in [12].

Theorem 46. Let $R_{1}, R_{2}$, and $R_{3}$ be any three hyperrings with strong epimorphisms $\phi_{i}: R_{i} \rightarrow R_{3}, i=1,2$, which are not strong isomorphisms. If $R_{1}$ and $R_{2}$ are hyperdomainlikes, then the pullback $P$ of $R_{1} \rightarrow R_{3} \leftarrow R_{2}$ is hyperdomainlike if and only if $\operatorname{Ker} \phi_{i} \subseteq Z\left(R_{i}\right), i=1,2$.

Proof. The proof is similar to the proof of Theorem 2.5 in [12].

Corollary 47. Let $R_{1}, R_{2}$, and $R_{3}$ be any three hyperrings with strong epimorphisms $\phi_{i}: R_{i} \rightarrow R_{3}, i=1,2$, which are not strong isomorphisms. If $R_{1}$ and $R_{2}$ are hyperdomainlikes, then the pullback $P$ of $R_{1} \rightarrow R_{3} \leftarrow R_{2}$ is associate but not hyperdomainlike if and only if $\operatorname{Ker} \phi_{i} \nsubseteq Z\left(R_{i}\right), i=1,2$, and $\phi_{1}\left(U\left(R_{1}\right)\right)=\phi_{2}\left(U\left(R_{2}\right)\right)$.

\section{Conflict of Interests}

The authors declare that there is no conflict of interests regarding the publication of this paper.

\section{Acknowledgment}

The authors would like to thank the referees for their critical reading of the paper and their suggestions.

\section{References}

[1] I. Kaplansky, "Elementary divisors and modules," Transactions of the American Mathematical Society, vol. 66, pp. 464-491, 1949.

[2] D. D. Anderson and S. Valdes-Leon, "Factorization in commutative rings with zero divisors," The Rocky Mountain Journal of Mathematics, vol. 26, no. 2, pp. 439-480, 1996.

[3] D. D. Anderson and S. Valdes-Leon, "Factorization in commutative rings with zero-divisors. II," in Factorization in Integral Domains, vol. 189 of Lecture Notes in Pure and Applied Mathematics, pp. 197-219, Marcle Dekker, New York, NY, USA, 1997.

[4] D. D. Anderson, M. Axtell, S. J. Forman, and J. Stickles, "When are associates unit multiples?" The Rocky Mountain Journal of Mathematics, vol. 34, no. 3, pp. 811-828, 2004.

[5] D. Spellman, G. M. Benkart, A. M. Gaglione et al., "Principal ideals and associate rings," JP Journal of Algebra, Number Theory and Applications, vol. 2, no. 2, pp. 181-193, 2002.

[6] M. Axtell, S. J. Forman, and J. Stickles, "Properties of domainlike rings," Tamkang Journal of Mathematics, vol. 40, no. 2, pp. 151164, 2009.
[7] A. Bouvier, "Anneaux présimplifiables et anneaux atomiques," Comptes Rendus Hebdomadaires des Séances de l'Académie des Sciences, Série A, vol. 272, pp. A992-A994, 1971.

[8] A. Bouvier, "Sur les anneaux de fractions des anneaux atomiques presimplifiables," Bulletin des Sciences Mathématiques, vol. 95, pp. 371-377, 1971.

[9] A. Bouvier, "Anneaux presimplifiables," Comptes Rendus de l'Académie des Sciences, vol. 274, pp. 1605-1607, 1972.

[10] A. Bouvier, "Resultats nouveaux sur les anneaux presimplifiables," Comptes Rendus de l'Académie des Sciences, vol. 275, pp. 955-957, 1972.

[11] A. Bouvier, "Anneaux presimplifiables," Revue Roumaine de Mathématique Pures et Appliquées, vol. 19, pp. 713-724, 1974.

[12] M. Ghanem, "Some properties of associate and presimplifiable rings," Turkish Journal of Mathematics, vol. 35, no. 2, pp. 333340, 2011.

[13] F. Marty, "Sur une generalization de la notion de groupe," in Proceedings of the 8th Congress Math Scandianaves, pp. 45-49, Stockholm, Sweden, 1934.

[14] J. Mittas, "Hypergroupes canoniques," Mathematica Balkanica, vol. 2, pp. 165-179, 1972.

[15] P. Corsini, Prolegomena of Hypergroup Theory, Aviain editore, Tricesimo, Italy, 2nd edition, 1993.

[16] P. Corsini and V. Leoreanu, Applications of Hyperstructure Theory, Advances in Mathematics, Kluwer Academic, Dordrecht, The Netherlands, 2003.

[17] B. Davvaz, Polygroup Theory and Related Systems, World Scientific, 2013.

[18] B. Davvaz and V. Leoreanu-Fotea, Hyperring Theory and Applications, International Academic Press, Palm Harbor, Fla, USA, 2007.

[19] T. Vougiouklis, Hyperstructures and Their Representations, vol. 115, Hadronic Press, Palm Harbor, Fla, USA, 1994.

[20] M. Krasner, "A class of hyperrings and hyperfields," International Journal of Mathematics and Mathematical Sciences, vol. 6, no. 2, pp. 307-311, 1983.

[21] R. Rota, "Strongly distributive multiplicative hyperrings," Journal of Geometry, vol. 39, no. 1-2, pp. 130-138, 1990.

[22] M. de Salvo, "Hyperrings and hyperfields," Annales Scientifiques de l'Université de Clermont-Ferrand II, vol. 22, pp. 89-107, 1984.

[23] B. Davvaz, "Isomorphism theorems of hyperrings," Indian Journal of Pure and Applied Mathematics, vol. 35, no. 3, pp. 321331, 2004.

[24] B. Davvaz, "Approximations in hyperrings," Journal of MultipleValued Logic and Soft Computing, vol. 15, no. 5-6, pp. 471-488, 2009.

[25] B. Davvaz and A. Salasi, "A realization of hyperrings," Communications in Algebra, vol. 34, no. 12, pp. 4389-4400, 2006.

[26] B. Davvaz and T. Vougiouklis, "Commutative rings obtained from hyperrings ( $H_{v}$-rings) with $\alpha^{*}$-relations," Communications in Algebra, vol. 35, pp. 3307-3320, 2007.

[27] S. Mirvakili, S. M. Anvariyeh, and B. Davvaz, "On $\alpha$-relation and transitivity conditions of $\alpha$," Communications in Algebra, vol. 36, no. 5, pp. 1695-1703, 2008.

[28] S. Mirvakili and B. Davvaz, "Applications of the $\alpha^{*}$-relation to Krasner hyperrings," Journal of Algebra, vol. 362, pp. 145-156, 2012.

[29] A. Nakassis, "Expository and survey article of recent results in hyperring and hyperfield theory," International Journal of Mathematics and Mathematical Sciences, vol. 11, pp. 209-220, 1988. 
[30] M. Stefănescu, "Constructions of hyperfields and hyperrings," Studii şi Cercetări Ştiinţifice, Seria: Matematică, Universitatea din Bacău, no. 16, supplement, pp. 563-571, 2006.

[31] T. Vougiouklis, "The fundamental relation in hyperrings. The general hyperfield," in Proceedings of the 4th International Congress on Algebraic Hyperstructures and Applications (AHA '91), pp. 203-211, World Scientific, 1991. 


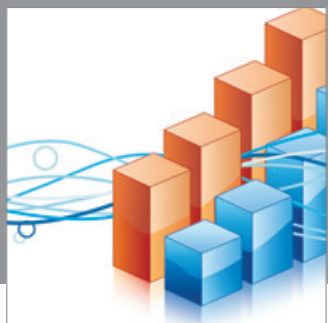

Advances in

Operations Research

mansans

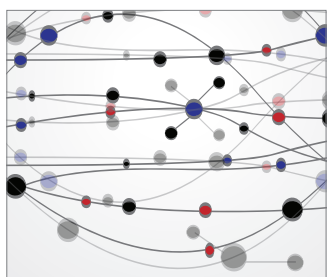

The Scientific World Journal
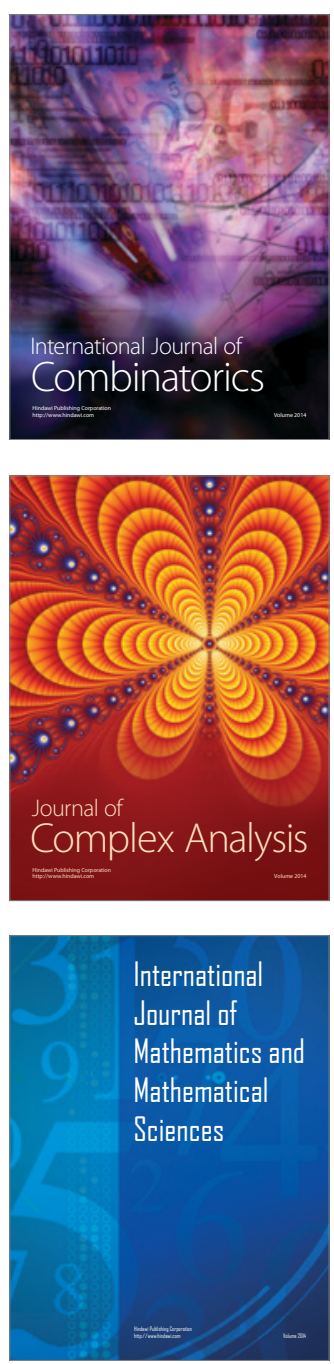
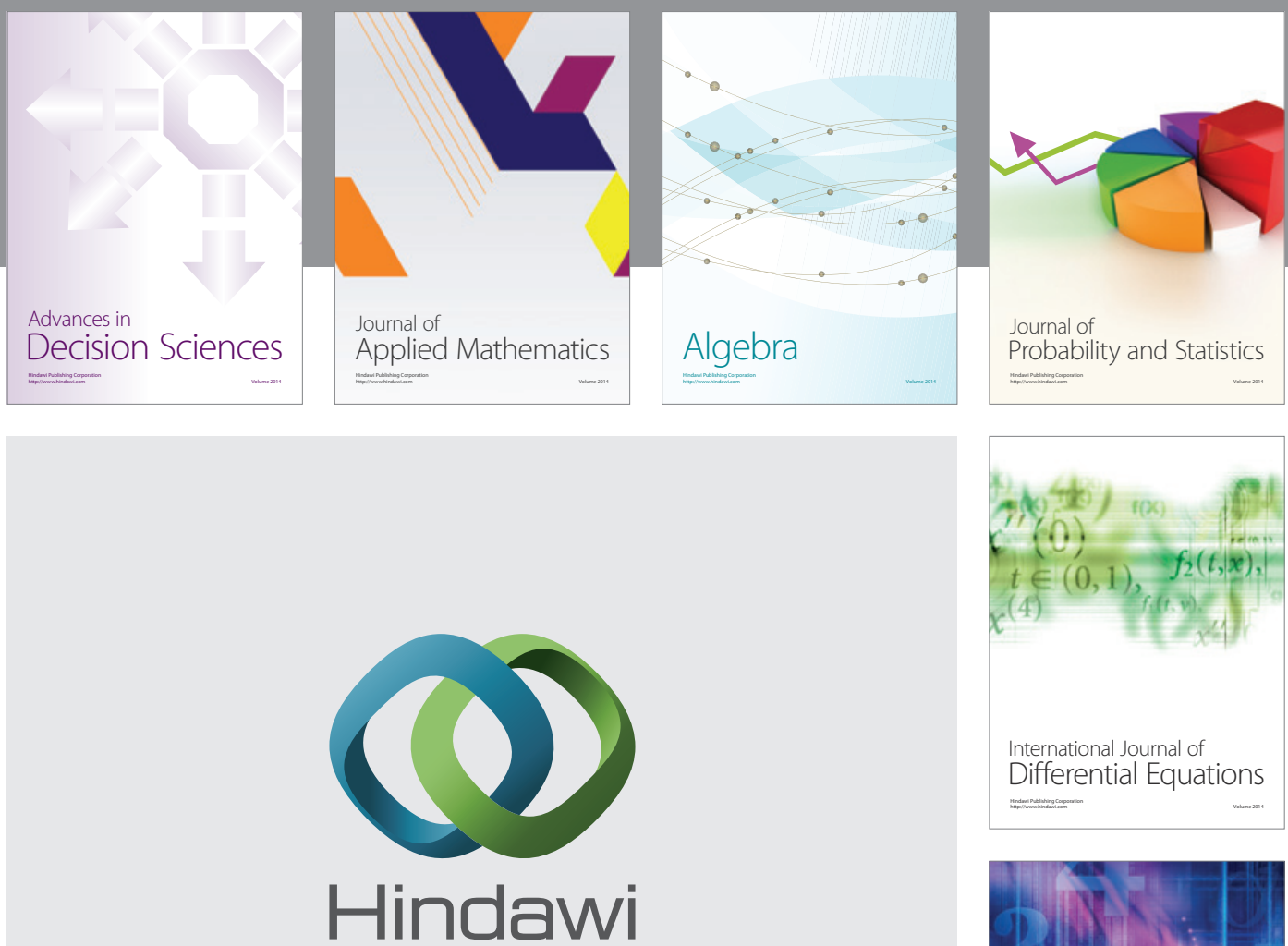

Submit your manuscripts at http://www.hindawi.com
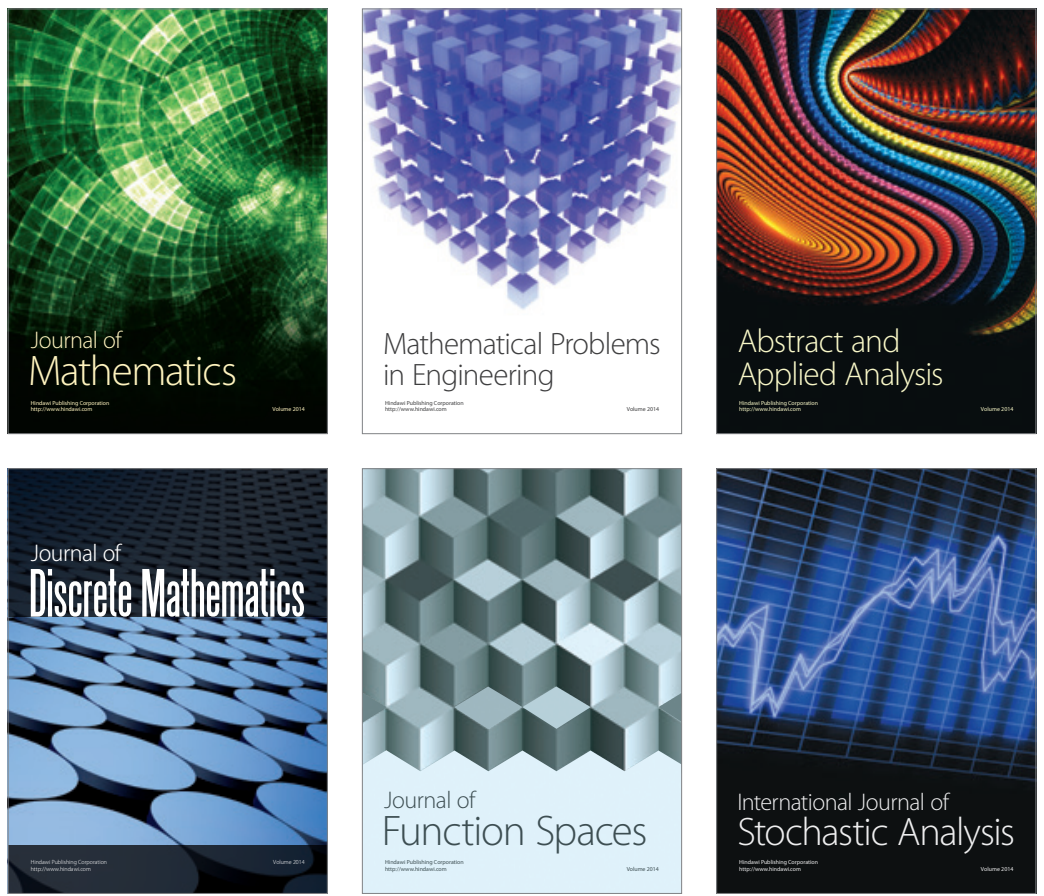

Journal of

Function Spaces

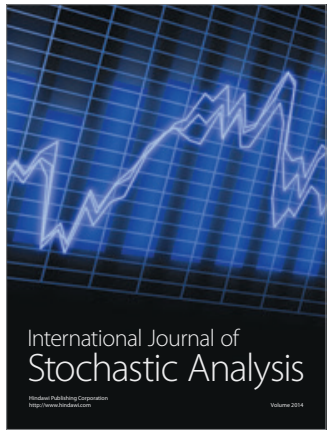

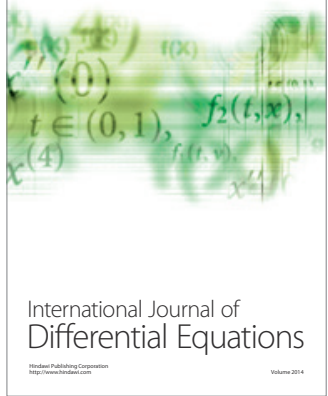
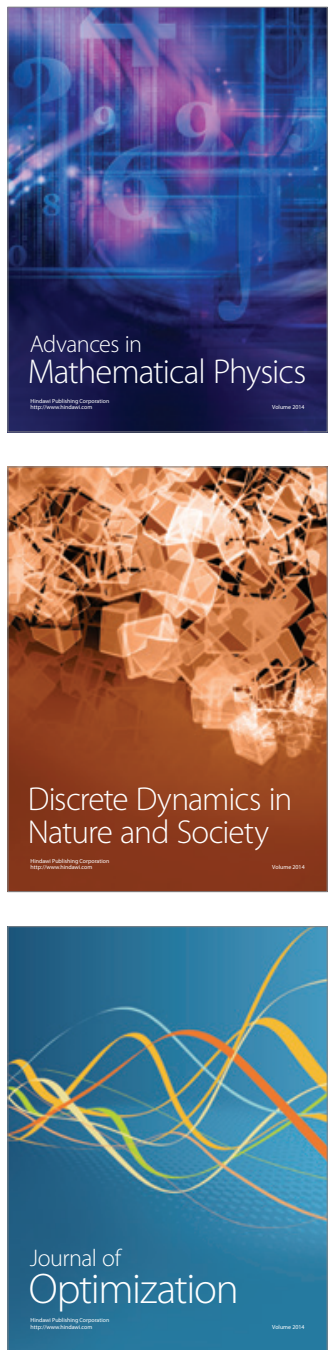